\title{
Effect of immediate versus early oral hydration on caesarean section postoperative outcomes: a randomized controlled trial
}

\author{
Heba A. Ahmed, Ahmed A. El-Shahawy, Hazem M. Sammour \\ Department of Obstetrics and Gynecology,Faculty of Medicine, Ain Shams University \\ Corresponding author: Heba A. Ahmed; Email: a.h_hoba@hotmail.com
}

\begin{abstract}
Background: Caesarean sections are increasing in number dramatically all over the world. So, it became very important to give more attention for their postoperative care. Objective: to evaluate the effect of immediate oral hydration initiated within 2 hours after uncomplicated Caesarean Section on the following post-operative outcomes: post-operative vomiting and abdominal distention, post-operative nausea and abdominal pain, the return of intestinal movements, duration of intravenous fluid administration, duration of hospital stay and participant satisfaction. Methods: this randomized controlled study was conducted at Ain Shams University Maternity Hospital. It was carried during the period from July 2016 to July 2017. 140 women, all of them underwent uncomplicated Cesarean Section under regional anesthesia were randomly assigned into two groups. In the immediate group: oral hydration was received in the first 2 hours postoperatively, and in the early group: oral hydration was received after 8 hours postoperatively. Results: all the results of the postoperative outcomes of both groups had non-significant differences except for the psychological satisfaction which was significantly higher in the immediate group with a $(\mathrm{p}<0.001)$. Conclusion: immediate oral hydration group showed non-significant differences comparing with early oral hydration group regarding most of the postoperative outcomes, but the results were relatively better towards the immediate group. Also, immediate hydration is significantly better than early hydration regarding psychological satisfaction of women, allowing them to be more able to breastfeed their kids and to spend a less stressful time in the hospital.
\end{abstract}

Key words: immediate/early oral hydration, cesarean section, postoperative outcomes, vomiting, nausea

\section{INTRODUCTION}

Caesarean sections performed every year are increasing in number dramatically all over the world. So, it became very important to give more attention for their postoperative care ${ }^{(\mathbf{1})}$.

Return of intestinal movement and passage of flatus are considered important factors affecting the duration of postoperative hospital stay in additional to other factors like the used anesthesia, wound healing, and breastfeeding ${ }^{(2)}$.

The traditional approach of giving the patient nothing orally postoperatively till bowel function returns (in the form of bowel movement or passage of flatus) followed by slow feeding is now challenged and became controversial ${ }^{(3)}$.

The importance of early feeding postoperatively depends on that food intake is able to stimulate a reflex causing a coordinated propulsive activity and increasing the secretion of gastrointestinal hormones. These effects cause an overall positive effect on intestinal movement decreasing the duration of postoperative ileus ${ }^{(4)}$. Early feeding also may be associated with reduced depletion of protein stores and improvement in wound healing with a faster recovery ${ }^{(5)}$.

There was a belief that postoperative oral intake without return of intestinal movements may cause nausea, vomiting, and abdominal distention leading to wound breakdown. However, this belief changed because the duration of surgery became shorter, the regional anesthesia became more available, and intestinal manipulation during surgery became minimal. All these factors provided the chance for early oral intake before bowel movements return ${ }^{(2)}$.

Several studies were done to compare between different regimens of postoperative hydration and feeding (2). Many of which were done on the effect of early hydration after Caesarean section, but less studies were done on the effect of immediate hydration.

Therefore, early oral hydration especially immediate supply of oral fluids after Caesarean sections needs further studying. Also, its impact on postoperative recovery and duration of stay in hospital needs to be studied widely as well. ${ }^{(2)}$

Moreover, early oral hydration after caesarean section might be beneficial in many ways, it may facilitate early return of intestinal movements and reduces the risk of hospital infections, leading to early hospital discharge ${ }^{(2)}$, it also may decrease the cost of hospital stay as it decreases the duration of intravenous fluid administration with less use of cannulae ${ }^{(3)}$. There is also a proof of its association with breastfeeding success and less suffering of the patient from thirst and hunger (6), early postoperative nutrition also leads to a better wound healing and a better muscle function with decreased sepsis ${ }^{(7)}$. 
Early fed women showed too higher levels of satisfaction, and early feeding wasn't associated with increased risk of paralytic ileus or gastrointestinal symptoms, it also didn't lead to increased incidence of postoperative complications like postoperative fever, wound dehiscence or wound sepsis ${ }^{(\mathbf{8})}$.

"Fast-track" surgery which is also called "Fast-track" rehabilitation is a multimodal concept for reducing general postoperative complications and for decreasing the length of hospital stay ${ }^{(9)}$.

Postoperative recovery speed is affected by some factors like the occurrence of pain, paralytic ileus, fatigue, sleep disturbances and postoperative nausea and vomiting. So, minimizing these factors through a multimodal approach is considered essential to enhance recovery ${ }^{(10)}$.

Good hydration is one of the methods to manage postoperative nausea and vomiting ${ }^{(\mathbf{1 1})}$. So, it is considered one of the elements of fast-track surgery.

\section{AIM OF THE WORK}

The aim of this study is to evaluate the effect of immediate oral hydration initiated within 2 hours after uncomplicated caesarean section on the following post-operative outcomes: postoperative vomiting and abdominal distention, postoperative nausea and abdominal pain, return of intestinal movements, duration of intravenous fluid administration, duration of hospital stay and participant satisfaction.

\section{PATIENTS AND METHODS}

Study design: A randomized controlled trial.

Study setting: This study was conducted at Ain Shams University Maternity Hospital; it was carried during the period from July 2016 to July 2017.

Participants: Pregnant women were recruited from those attended Ain Shams Maternity Hospital and scheduled for Cesarean Section.

\section{Selection of the participants:}

Inclusion criteria: Uncomplicated pregnancy. Uncomplicated elective or emergency Caesarean section under regional anesthesia. Level of hemoglobin is not less than $10 \mathrm{~g} / \mathrm{dl}$. BMI $<30$ $\mathrm{kg} / \mathrm{m}^{2}$.

Exclusion criteria: Medical disorders in the form of HTN, DM, and Hepatic disorders. Bleeding disorders. Intraoperative bowel or bladder injury during the cesarean section. Intraoperative or immediate postoperative severe bleeding. Chronic gastrointestinal problems, like chronic constipation, peptic ulcer, oesophagitis, hiatus hernia or irritable bowel syndrome. Severe abdominal adhesions.

Out of 173 women who underwent Cesarean Section under spinal anesthesia and matched the inclusion criteria, 20 women refused to agree on consent to participate in the study, 12 women were excluded because they had medical disorders like DM and hypertension, one was excluded because she had immediate postoperative severe bleeding.

So, this study included 140 women, all of them underwent uncomplicated Cesarean Section under regional anesthesia, they were randomly assigned into two groups: Group I (experimental group " Immediate hydration") "70 women": These women received clear fluids in the first 2 hours postoperatively. Group E (control group "Early hydration") "70 women": And these women received clear fluids after 8 hours postoperatively.

Sample Size calculation: The required sample size has been calculated using the Power Analysis and Sample Size Software (PASS ()) version 11.0.10 (NCSS $\odot$, LLC. Kaysville, Utah, USA). The primary outcome is the occurrence of postpartum abdominal distension post surgery until the time of hospital discharge. A previous study reported that the rate of occurrence of abdominal distension in group A (Early Feeding) was $4.28 \%$; while it was $20.0 \%$ in group B (Late Feeding) ${ }^{\mathbf{1 2})}$. So, it was estimated that Group sample sizes of 70 in group one (Early feeding) and 70 (Late feeding) in group two achieve $80 \%$ power to detect a difference between the group proportions of 0.1580 . The proportion in group one (the treatment group) is assumed to be 0.2000 under the null hypothesis and 0.0420 under the alternative hypothesis. The proportion of group two (the control group) is 0.2000 . The test statistic used is the two-sided $\mathrm{Z}$ test with pooled variance. The significance level of the test was targeted at 0.0500 . The significance level achieved by this design is 
0.0506. $15 \%$ lost to follow up were taken into consideration while calculating sample size.

\section{Randomization and allocation:}

Randomization: It was performed by computer-generated random sequence (MicrosoftSeattle, WA, USA).

Allocation concealment: By using sealed opaque sequentially numbered envelopes, each envelope included the type of intervention: If the letter inside the envelope was "I" which means "immediate hydration", the woman was given a bottle of water to start drinking it. After about 1 or 2 hours she was offered a cup of clear warm fluid like peppermint or anise, she was allowed to drink whatever she wants after that according to her needs (but not milk or soda containing drinks). While if the letter inside the envelope was " $E$ " which means "early hydration": the woman was allowed to drink after 8 hours. Both the caregivers and the participants were blinded to the group allocation.

\section{Ethical and legal aspects:}

1. Patient information and informed consent: A written informed consent in the Arabic language was signed by each woman explaining the nature, scope and possible consequences of the clinical study. The woman's consent was confirmed by the personally dated signature of the woman and personally dated signature of the person conducting the informed consent.

2. Study approval: This study was approved by the ethical and research committee of the council of obstetrics and gynecology department, Ain Shams University.

\section{The study methods:}

1. Personal data and written consent: After the woman reaches the recovery room, some demographic data were taken from her like age, parity, gestational age and cause of Cesarean Section. Then the study with its nature, benefits, and risks was explained to the woman, and written consent in the Arabic language of her agreement to be enrolled in the study was taken, that consent was confirmed by personally dated signatures of both the woman and the person who conducted the consent.

2. History taking: The following histories were revised from the admission sheet and the woman herself to make sure that all inclusion criteria were met and to exclude all the exclusion criteria:
Operative history: to make sure of undergoing the operation under regional anesthesia, the operation was done by same rank senior residents with almost the same technique, and the operation time ranged from 30-50 minutes. Also, to exclude intraoperative complications (like bowel or bladder injury), severe intraoperative bleeding, and severe intestinal adhesions that may interfere with bowel movement return after the operation.

Medical history: to exclude: hypertension, diabetes mellitus, hepatic diseases, bleeding disorders, chronic gastrointestinal problems and anemia $<10 \mathrm{mg} / \mathrm{dl}$.

Obstetric history: to make sure that her pregnancy was not complicated.

Also, the weight and the length of the woman were checked to make sure that her BMI wasn't exceeding 30 if she was suspected to be overweight.

3. Randomization: After Cesarean Section was done, each woman had a numbered opaque envelope which was opened just after the operation to indicate the assigned group.

4. Oral fluids regimens: For the "immediate hydration" group: the woman was given a bottle of water to start drinking it. After about 1 or 2 hours she was offered a cup of clear warm fluid like peppermint or anise, she was allowed to drink whatever she wants after that according to her needs (but not milk or soda containing drinks). For the "early hydration" group: the woman was allowed to drink after 8 hours. For both groups, when the oral fluids were well tolerated, semisolid and solid foods were allowed gradually.

5. Intravenous fluids regimen and analgesia: All women were given the same hospital intravenous fluid regimen which was 500 $\mathrm{ml}$ of $5 \%$ glucose every 6 hours, $500 \mathrm{ml}$ of Ringer lactate every 12 hours and $500 \mathrm{ml}$ of normal saline every 24 hours. Taking into consideration that these intravenous fluids were discontinued when the bowel movements started early and the woman feeding was well established before 24 hours. All women received the same routine analgesia that was added to the first bottle of intravenous fluids given to them in the recovery room; it was Ketolak $30 \mathrm{mg}$ IV injection/12 hours. 
6. Data collection about the primary outcomes: While the woman was in the recovery room and after transferring her to the postpartum ward, the following data were collected by the caregivers after excluding postpartum hemorrhage:

The occurrence of vomiting and abdominal distention: These data were taken from women every 1 hour in the $1^{\text {st }} 2$ hours, then every 2 hours until the time of hospital discharge (depending on that 0 hours is the onset of surgery end).

7. Data collection about the secondary outcomes: The occurrence of nausea and abdominal pain: Similar to the primary data, these data were collected from women every 1 hour in the $1^{\text {st }} 2$ hours, then every 2 hours until the time of hospital discharge. The degree of pain was determined according to the visual analog scale VAS which includes 5 points verbal descriptive scale (nil, mild, moderate, severe and very severe) and by using a simple questionnaire with recording the pain beginning from the moderate degree.

Return of intestinal movements: And this was done by recording the timing of: Hearing of the $\mathbf{1}^{\text {st }}$ intestinal sound: Abdominal auscultation was done 2 hours postoperatively and every 2 hours to detect intestinal sounds, taking into consideration that the participants didn't receive any antiemetics or laxatives routinely during the follow-up period.

Passage of flatus: The woman was asked about that every 2 hours postoperatively. Duration of intravenous fluids administration: And this was by recording the timing of discontinuation of intravenous fluids. Initiation of breastfeeding: Its timing was recorded too. Duration of hospital stay: It was calculated by recording the timing of hospital discharge. The routine hospital stay period (from the surgery onset until the hospital discharge) ranges between $24-48$ hours.

Hospital discharge was done when the following criteria were met: tolerance of the regular diet without nausea or vomiting, return of intestinal movements, breastfeeding success, normal vital signs, ability to ambulate and urinate without assistance, and absent unresolved postoperative complications.

8. A questionnaire about psychological satisfaction: Psychological satisfaction was one of the secondary outcomes to be reported. So, before the woman discharge from the hospital, she was asked about her psychological satisfaction regarding the timing of hydration initiation according to the group she was enrolled in, and if she was planning to repeat the same oral hydration timing in the following deliveries.

\section{Statistical Methods:}

Data were analyzed using Stata ${ }^{\circledR}$ version 14.2 (StataCorp LLC, College Station, TX, USA).

Normality of numerical data distribution was examined using the Shapiro-Wilk test. Normally distributed numerical variables were presented as the mean and standard deviation (SD) and intergroup differences were compared using the independent-samples t-test.

Categorical data were presented as number and percentage and differences were compared using Fisher's exact test (for nominal data) or the chi-squared test for trend (for ordinal data).

Time to event analysis was done using the Kaplan-Meier method. The log-rank test was used to compare Kaplan-Meier curves.

P-values $<0.05$ were considered statistically significant.

\section{RESULTS}

In the current study, women who had medical disorders in the form of HTN, DM, and Hepatic disorders were excluded from the study, along with women who had bleeding disorders, intraoperative bowel or bladder injury, or intraoperative or immediate postoperative severe bleeding. Having chronic gastrointestinal problems like chronic constipation, peptic ulcer, oesophagitis, hiatus hernia or irritable bowel syndrome and having severe abdominal adhesions were exclusion criteria too.

Regarding the results of the study, it was interesting to show that there were "no" significant differences between the two groups regarding the primary postoperative outcomes which were the frequency of vomiting and abdominal distention. Also, there were no significant differences found between both groups regarding most of the secondary postoperative outcomes like the frequency of nausea and abdominal pain, time until return of intestinal movements, time of passage of flatus, time to initiate breastfeeding, timing until removal of intravenous fluids and time of hospital discharge.

The only highly significant difference between both groups was regarding psychological 
satisfaction with the p-value $<0.001$. Most of the women in the immediate oral hydration group were satisfied with the method of hydration as they were not experiencing the thirst sensation after the operation allowing them to be less stressful, and they decided to choose the immediate hydration again in the following deliveries.

Both groups were matched regarding maternal age, gestational age, parity and indications of Cesarean Section.

Although the difference between the two groups was not significant regarding most of the study results, the immediate oral hydration group showed many results that were relatively better than the early oral hydration group. The time until the return of intestinal sounds in the immediate hydration group $(6.7 \pm 3.3$ hours) was shorter than the early hydration group (7.5 \pm 3.7 hours). Also, the time until the passage of flatus in the immediate hydration group (11.2 \pm 6.5 hours) was shorter than the early hydration group (11.6 \pm 5.2 hours). Immediate hydration group showed less frequent nausea, vomiting and abdominal pain than the early hydration group. Both groups were equal regarding the incidence of abdominal distention.

Also, the time until discontinuation of intravenous fluids was shorter in the immediate hydration group (14.6 \pm 4.4 hours) compared with the early hydration group $(15.3 \pm 3.5$ hours $)$. The same was regarding the time to initiate breastfeeding which was shorter in the immediate oral hydration group (3.4 hours) compared with the early hydration group (4.2 hours).

The time until hospital discharge was shorter in the early hydration group $(30.1 \pm 7.9$ hours) compared with the immediate hydration group (31.2 \pm 10.7 hours) with a nonsignificant difference. But overall, the hospital discharge policy in the hospital was not related directly to the results of the study; it depended on that the woman should stay at least 24 hours in the hospital before discharge and after making sure that she met the discharge criteria.

There was a highly significant difference between the two groups regarding psychological satisfaction in favor of the immediate group with the $\mathrm{p}$ value $<0.001$. In the immediate oral hydration group, 64 (91.4\%) women reported they were satisfied, 3 (4.3\%) women were neutral, while $3(4.3 \%)$ women were dissatisfied. In the early oral hydration group, 40 (57.1\%) women reported they were satisfied, $3(4.3 \%)$ women were neutral, while 27 (38.6\%) women were dissatisfied. We considered this result a very important one as the psychological satisfaction allows the woman to be less stressful and to enjoy her stay in the hospital more, with a better ability to breastfeed her baby.

Table (1): Demographic characteristics of both study groups.

\begin{tabular}{|c|c|c|c|}
\hline Variable & $\begin{array}{c}\text { Immediate } \\
\text { hydration }(\mathbf{n = 7 0 )}\end{array}$ & $\begin{array}{c}\text { Early } \\
\text { hydration } \\
(\mathbf{n = 7 0 )}\end{array}$ & p-value \\
\hline Age (years) & $28.9 \pm 5.4$ & $27.4 \pm 5.2$ & $0.090 \mathrm{II}$ \\
\hline $\begin{array}{c}\text { Gestational age } \\
\text { (weeks) }\end{array}$ & $38.2 \pm 1.7$ & $37.7 \pm 1.8$ & $0.084 \mathbb{I}$ \\
\hline Parity & & & $0.182 \S$ \\
\hline P1 & $12(17.1 \%)$ & $14(20.0 \%)$ & \\
\hline P2 & $22(31.4 \%)$ & $26(37.1 \%)$ & \\
\hline P3 or higher & $36(51.4 \%)$ & $30(42.9 \%)$ & \\
\hline $\begin{array}{c}\text { Indication for } \\
\text { CS }\end{array}$ & & & $0.078 ¥$ \\
\hline Previous CS & $47(67.1 \%)$ & $47(67.1 \%)$ & \\
\hline Fetal distress & $15(21.4 \%)$ & $12(17.1 \%)$ & \\
\hline Malpresentation & $2(2.9 \%)$ & $9(12.9 \%)$ & \\
\hline Others & $6(8.6 \%)$ & $2(2.9 \%)$ & \\
\hline
\end{tabular}

Data are mean \pm SD or number $(\%) . \quad \mathrm{CS}=$ Cesarean Section. $\mathrm{P} 1, \mathrm{P} 2, \mathrm{P} 3=$ para1, para2, para3

Table 1 shows the demographic characteristics of both study groups; they have insignificant differences.

Table (2): Time to main end-points in both study groups.

\begin{tabular}{|l|l|c|c|c|}
\hline Variable & \multicolumn{1}{|c|}{ End-point } & $\begin{array}{c}\text { Immediate } \\
\text { hydration } \\
(\mathrm{n}=70)\end{array}$ & $\begin{array}{c}\text { Early } \\
\text { hydration } \\
(\mathrm{n}=70)\end{array}$ & p-valuefl \\
\hline \multirow{4}{*}{$\begin{array}{l}\text { Time } \\
\text { (h) }\end{array}$} & $\begin{array}{l}\text { Recovery of } \\
\text { intestinal sounds }\end{array}$ & $6.7 \pm 3.3$ & $7.5 \pm 3.7$ & 0.205 \\
\cline { 2 - 5 } & Passage of flatus & $11.2 \pm 6.5$ & $11.6 \pm 5.2$ & 0.708 \\
\cline { 2 - 5 } & $\begin{array}{l}\text { Discontinuation of } \\
\text { IV fluids }\end{array}$ & $14.6 \pm 4.4$ & $15.3 \pm 3.5$ & 0.296 \\
\cline { 2 - 5 } & Hospital discharge & $31.2 \pm 10.7$ & $30.1 \pm 7.9$ & 0.495 \\
\hline
\end{tabular}

Data are mean $\pm \mathrm{SD} . \mathrm{h}=$ hour.

Table 2 shows there were insignificant difference between both groups regarding these postoperative outcomes.

Table (3): Incidence of adverse outcomes in both study groups.

\begin{tabular}{|l|c|c|c|}
\hline $\begin{array}{c}\text { Adverse } \\
\text { outcome }\end{array}$ & $\begin{array}{c}\text { Immediate } \\
\text { hydration }(\mathbf{n}=\mathbf{7 0})\end{array}$ & $\begin{array}{c}\text { Early hydration } \\
(\mathbf{n = 7 0})\end{array}$ & p-valueIl \\
\hline Nausea & $1(1.4 \%)$ & $2(2.9 \%)$ & 1.000 \\
\hline Vomiting & $0(0.0 \%)$ & $1(1.4 \%)$ & 1.000 \\
\hline Distention & $6(8.6 \%)$ & $6(8.6 \%)$ & 1.000 \\
\hline Pain & $5(7.1 \%)$ & $7(10.0 \%)$ & 0.563 \\
\hline
\end{tabular}

Data are number $(\%)$. 
Table 3 shows there were insignificant difference between both groups regarding these postoperative outcomes too.

Table (4): Women satisfaction in both study groups.

\begin{tabular}{|c|l|c|c|c|}
\hline Variable & Rating & $\begin{array}{c}\text { Immediate } \\
\text { hydration } \\
(\mathbf{n}=\mathbf{7 0})\end{array}$ & $\begin{array}{c}\text { Early } \\
\text { hydration } \\
(\mathbf{n = 7 0})\end{array}$ & $\begin{array}{c}\text { p- } \\
\text { value } \\
\mathbb{I}\end{array}$ \\
\hline \multirow{2}{*}{$\begin{array}{l}\text { Patient } \\
\text { satisfaction }\end{array}$} & $\begin{array}{l}\text { Dissatisf } \\
\text { ied }\end{array}$ & $3(4.3 \%)$ & $27(38.6 \%)$ & $<0.00$ \\
\cline { 2 - 5 } & Neutral & $3(4.3 \%)$ & $3(4.3 \%)$ & \\
\cline { 2 - 5 } & Satisfied & $64(91.4 \%)$ & $40(57.1 \%)$ & \\
\hline
\end{tabular}

Data are number (\%).

Table 4 shows there was a statistically significant difference between both groups ( $\mathrm{p}$-value $<0.001)$.

\section{DISCUSSION}

All over the world, cesarean sections performed yearly are dramatically increasing in number. Therefore, it became very important to give more attention to their postoperative care ${ }^{(5)}$.

The traditional approach of giving the patient nothing orally postoperatively till bowel function returns (in the form of bowel movement or passage of flatus) followed by slow feeding is now challenged and became controversial ${ }^{(3)}$.

Many previous studies discussed the effect of early oral hydration after Cesarean Section like Mulayim et al. (2) who included in his study 200 women, Ghareeb et al. ${ }^{(3)}$ who included 40 women, Jalilian et al. ${ }^{\left({ }^{9}\right)}$ who included 140 women in his study. These studies initiated oral hydration after 2 hours postoperatively for half of the participants (early hydration), while the other half initiated oral hydration after 8 hours postoperatively (delayed hydration), and all of them underwent Cesarean Section under regional anesthesia.

The literature results from these three studies were in agreement with our findings in having no significant differences between both the participating groups regarding the incidence of nausea and vomiting. Our study was also similar to Mulayim $\boldsymbol{e t}$ al. ${ }^{(2)}$ in that there was no significant difference between both groups regarding the incidence of abdominal distention and abdominal pain.

Whereas, the results of these three studies were different from our study in indicating that there was a significant difference between the two groups regarding the timing till bowel movement which was shorter in the early hydration group than the delayed hydration one, while in our study the difference wasn't significant. Ghareeb et al. ${ }^{(3)}$ study was different regarding the length of hospital stay which was shorter in the early hydration group with a significant difference, while this difference wasn't significant in our study too. The incidence of abdominal distention in Jalilian et al. ${ }^{(\mathbf{1 2})}$ was less in the early group with a significant difference, while this difference wasn't significant in our study between both groups.

Only a few studies discussed the effect of immediate oral hydration within the first 2 hours postoperatively like Guedj $\boldsymbol{e t}$ al. ${ }^{(13)}$ and $\mathrm{Abd}$ Rabbo ${ }^{(14)}$

The results of our study were very similar to the results of Guedj et al. ${ }^{(13)}$ which showed that there were no significant differences between both groups regarding postoperative outcomes and intestinal movements, but there was much better comfort among the women of immediate oral hydration group. Guedj et al. ${ }^{(13)}$ included in his study 53 women underwent Cesarean section under epidural anesthesia (29 of them received immediate oral hydration while 22 received oral hydration after 24 hours postoperatively) and this low sample size was a weak point. Women of the immediate group didn't receive any intravenous fluids besides oral hydration in his study, and this was a good point.

Our results went on the same track with Abd Rabbo ${ }^{(14)}$ who included in their study 200 women underwent Cesarean section under general anesthesia "not regional," 100 (oral hydration immediately) and 100 (oral hydration after 24 hours). His results showed that the immediate group was better than the late group in everything like faster bowel movement return, faster breastfeeding, less nausea and vomiting and shorter time of hospital stay. The difference between both groups in his study was significant while in our study it was not.

\section{CONCLUSION}

Immediate oral hydration for women who underwent uncomplicated Cesarean Section under regional anesthesia resulted in non-significant differences comparing with early oral hydration regarding most of the postoperative outcomes. But the results were relatively better towards the immediate group like less frequent postoperative nausea, vomiting, and abdominal pain, shorter time until return of bowel movement and faster initiation of breastfeeding and removal of intravenous fluid. 
Also, immediate oral hydration is significantly better than early hydration regarding psychological satisfaction of women, allowing them to be more able to breastfeed their kids and to spend a less stressful time in the hospital.

\section{RECOMMENDATIONS}

Immediate oral hydration after uncomplicated Cesarean Sections under regional anesthesia should be used more widely, and it should be given more attention as one of the elements of fast-track surgeries. Further studies needed to confirm the results of this study and to evaluate the effect of immediate oral hydration regarding more postoperative outcomes.

\section{REFERENCES}

1. Malhotra N, Khanna S, Pasrija Sh, Jain M and Agarwala R (2005): Early oral hydration and its impact on bowl activity after elective Caesarean section. European Journal of Obstetrics, Gynecology and Reproductive Biology, 120: 53-56.

2. Mulayim B, Celic N, Kaya $S$ and Yanik $\mathbf{F}$ (2008): Early oral hydration after Caesarean delivery performed under regional Anesthesia. International Journal of Gynecology and Obstetrics, 101: 273-276.

3. Ghareeb S, Ahmad E and Turki H (2013): Effect of early oral hydration on post Caesarean outcomes. Journal of American science, 9(8):70-78.

4. Michael D, Johnson, MD, R. Matthew Walsh, MD (2009): Current therapies to shorten postoperative ileus. Cleveland Clinic Journal of Medicine, 76(11): 641-648.

5. Betrán AP, Ye J, Moller AB, Zhang J, Gülmezoglu AM and Torloni MR (2016): The increasing trend in caesarean section rates: global, regional and national estimates: 19902014. PloS One, 11(2):e0148343.

6. Khawaja M, Jurdi R and Kabakian-Khasholian $T$ (2004): Rising trends in cesarean section rates in Egypt. Birth, 31(1):12-6.

7. Kawasaki N, Suzuki Y, Nakayoshi T, Hanyu N, Nakao M, Takeda A, Furukawa Y and Kashiwagi H. (2009): Early postoperative enteral nutrition is useful for recovering gastrointestinal motility and maintaining the nutritional status. Surgery Today, 39(3):225230.
8. Ogbadua AO, Agida TE, Akaba GO, Akitoye OA and Ekele BA (2018): Early Versus Delayed Oral Feeding after Uncomplicated Cesarean Section under Spinal Anesthesia: A Randomized Controlled Trial. Nigerian Journal of Surgery, 24(1):6-11.

9. Schwenk W, Muller JM (2005): What is "fast-track" surgery?. Dtsh med Wochenschr, 130(10): 536-540.

10. Henrik K, Douglas W (2008): EvidenceBased Surgical Care and the Evolution of FastTrack Surgery. Annals of Surgery, 248(2): 189-198.

11. Kehlet H, Wilmore DW (2002): Multimodal strategies to improve surgical outcome. Am J Surg., 183:630-41.

12. Jalilian N, Fakhri $M$ and Keshavarzi $F$ (2013): A randomized clinical trial to compare the post-operative outcomes of early vs. late oral feeding after Cesarean section. Life Science Journal, 10(8s):212-215

13. Guedj P, Eldor $J$ and Stark $M$ (1991): Immediate postoperative oral hydration after caesarean section. Asia-Oceania Journal of Obstetrics and Gynaecology, 17(2):125-9.

14. Abd Rabbo S (1995): Early oral hydration: A novel regimen for management after elective Cesarean Section. Journal of obstetrics and gynaecology, 21(6):563-567. 